\title{
Feebly compact paratopological groups and real-valued functions
}

\author{
Manuel Sanchis · Mikhail Tkachenko
}

This is a preliminary version of the article published in Monatsh. Math. 168 no. 3 (2012), 579-597.

\begin{abstract}
We present several examples of feebly compact Hausdorff paratopological groups (i.e., groups with continuous multiplication) which provide answers to a number of questions posed in the literature. It turns out that a 2-pseudocompact, feebly compact Hausdorff paratopological group $G$ can fail to be a topological group. Our group $G$ has the Baire property, is Fréchet-Urysohn, but it is not precompact.

It is well known that every infinite pseudocompact topological group contains a countable non-closed subset. We construct an infinite feebly compact Hausdorff paratopological group $G$ all countable subsets of which are closed. Another peculiarity of the group $G$ is that it contains a nonempty open subsemigroup $C$ such that $C^{-1}$ is closed and discrete, i.e., the inversion in $G$ is extremely discontinuous.

We also prove that for every continuous real-valued function $g$ on a feebly compact paratopological group $G$, one can find a continuous homomorphism $\varphi$ of $G$ onto a second countable Hausdorff topological group $H$ and a continuous real-valued function $h$ on $H$ such that $g=h \circ \varphi$. In particular, every feebly compact paratopological group is $\mathbb{R}_{3}$-factorizable. This generalizes a theorem of Comfort and Ross established in 1966 for real-valued functions on pseudocompact topological groups.
\end{abstract}

Keywords Paratopological group · Feebly compact · 2-pseudocompact · precompact $\cdot$ Regularization $\cdot \mathbb{R}_{3}$-factorizable

Mathematics Subject Classification (2000) 22A30 - 54H11 · 54C30

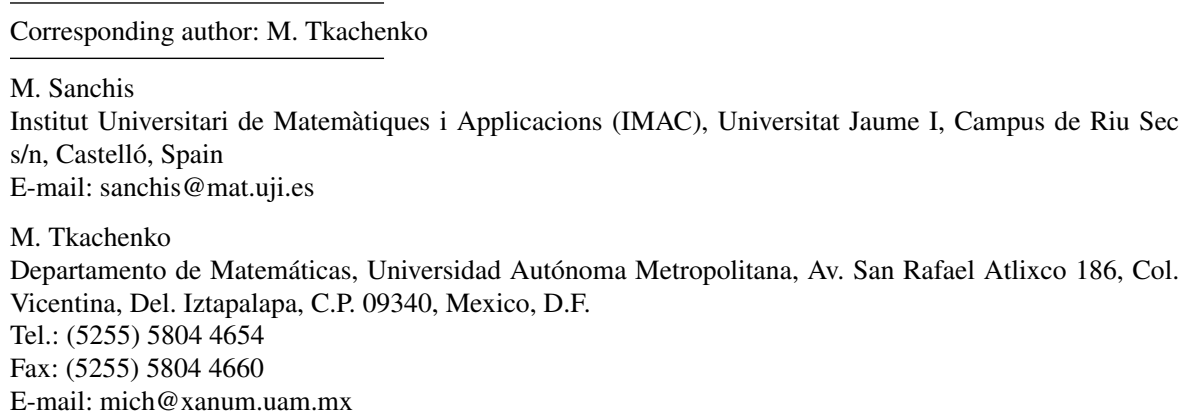




\section{Introduction}

One of the first results on the automatic continuity of multiplication and inversion in semitopological groups was obtained by Montgomery in [20]. He proved that every completely metrizable semitopological group $G$ (a group with separately continuous multiplication) is in fact a paratopological group, i.e., multiplication in $G$ is jointly continuous. In addition, if $G$ is separable, then the inversion in $G$ is also continuous and $G$ is a topological group. In 1960, Żelazko [36] eliminated the assumption about the separability of $G$ in the latter fact by showing that every completely metrizable semitopological group is a topological group.

Compactness has an even stronger impact on automatic continuity of operations in Topological Algebra than complete metrizabilty. By the celebrated theorem of Numakura in [21], every compact Hausdorff topological semigroup with two-sided cancellation is a topological group. In particular, compact Hausdorff paratopological groups are topological groups. The latter fact has been considerably generalized by Ellis, Grant, Brand, Bouziad, Bokalo and Guran, Romaguera and Sanchis, KenderovKortezov-Moors, and some others (see [11,15,9,8,7,30,18]).

The next step in the study of automatic continuity in paratopological groups was done by Reznichenko in [28] where he proved that every pseudocompact paratopological group $G$ is a topological group, i.e., the inversion in $G$ is continuous. In [2], Arhangel'skii and Reznichenko extended this result to regular pseudocompact paratopological groups. Since the term 'pseudocompact' is usually reserved for Tychonoff spaces, we will replace it with 'feebly compact' when referring to regular or Hausdorff spaces. Thus, a space is called feebly compact if every locally finite family of open sets in the space is finite. It is clear that feeble compactness and pseudocompactness coincide in the class of completely regular spaces.

It was recently shown by Ravsky in [23, Example 3] that there exists a feebly compact Hausdorff paratopological group which fails to be a topological group. In other words, Arhangel'skii-Reznichenko's theorem cannot be extended to Hausdorff paratopological groups. Furthermore, under Martin's Axiom, there exists a countably compact Hausdorff paratopological group with discontinuous inversion [25, Example 2].

In this article we continue the study of pseudocompact and 2-pseudocompact paratopological groups originated in [1,2,25,27, 28,30]. The authors of [1] and [25] presented several conditions which, combined with feeble compactness or 2-pseudocompactness, imply that a paratopological group is in fact a topological group. For example, every feebly compact periodic Hausdorff paratopological group is a topological group according to [25, Proposition 4], while [25, Proposition 5] implies that every 2-pseudocompact paratopological group of countable pseudocharacter is also a topological group.

Rephrasing Question A from [1], Ravsky asked in [25] if every 2-pseudocompact Hausdorff paratopological group was a topological group. Since countable compactness implies 2-pseudocompactness in paratopological groups, it follows from [25, Example 2] that, consistently, the answer to Ravsky's question is "no". We answer this question in Theorem 1 in the negative by constructing in $Z F C$ an example of a 2-pseudocompact, feebly compact Hausdorff paratopological group $G$ that fails to be 
a topological group. Our group $G$ has the Baire property but it is not precompact, so Theorem 1 provides an answer (again in the negative) to the third item of Problem 1 in [25]. It is worth mentioning that the group $G$ is Fréchet-Urysohn and the product $G \times X$ is feebly compact for every feebly compact space $X$ (see Remark 1 ).

It is well known that every infinite precompact topological group $G$ contains a countable non-closed subset-it suffices to take a countably infinite subgroup $C$ of $G$ and put $A=C \backslash\{e\}$, where $e$ is the identity of $G$. Then $A$ cannot be closed in $G$ since otherwise $C$ would be a discrete subgroup of $G$ and both $C$ and $G$ would not be precompact. The same conclusion is valid for pseudocompact topological groups since they are precompact by [10, Theorem 1.1]. In Theorem 2 we present an example of an infinite feebly compact Hausdorff paratopological group $G$ all countable subsets of which are closed. The peculiarity of the group $G$ is that it contains a nonempty open subsemigroup $C$ such that $C^{-1}$ is a closed discrete subset of $G$, i.e., the inversion in $G$ is "extremely discontinuous". It is clear that any paratopological group with this combination of properties cannot be 2-pseudocompact. Nevertheless, in Theorem 3 we construct a feebly compact, 2-pseudocompact Hausdorff paratopological group $H$ such that all countable subsets of $H$ are closed.

In Section 3 we consider continuous real-valued functions on feebly compact paratopological groups. No separation axioms are imposed on the groups there. We prove in Theorem 5 that for every continuous real-valued function $g$ on a feebly compact paratopological group $G$ one can find a continuous homomorphism $\varphi$ of $G$ onto a second countable Hausdorff topological group $H$ and a continuous real-valued function $h$ on $H$ such that $g=h \circ \varphi$. In particular we see that every feebly compact paratopological group is $\mathbb{R}_{3}$-factorizable. This result extends a similar property of continuous real-valued functions on pseudocompact topological groups (see [10, Theorem 1.5]).

By a theorem in [10], every pseudocompact topological group is precompact, while Theorem 3.8 from [34] implies that all precompact topological groups are $\mathbb{R}$ factorizable. Somewhat surprisingly, Theorem 5 is not valid for precompact paratopological groups, even if one considers only Tychonoff paratopological groups. Once again, this result shows the big difference in the properties of the two classes of objects of Topological Algebra.

\subsection{Notation and terminology}

A space $X$ is said to be feebly compact if every locally finite family of open sets in $X$ is finite. In the class of Tychonoff spaces, feeble compactness is equivalent to pseudocompactness.

A sequence $\left\{U_{n}: n \in \omega\right\}$ of subsets of a space $X$ is decreasing if $U_{n} \supset U_{n+1}$ for each $n \in \omega$. A paratopological group $G$ is 2-pseudocompact if $\bigcap_{n=0}^{\infty} c l_{G} U_{n}^{-1} \neq \emptyset$ for each decreasing sequence $\left\{U_{n}: n \in \omega\right\}$ of nonempty open subsets of $G$. Clearly, every countably compact paratopological group is 2-pseudocompact.

A paratopological group $G$ is precompact if for every neighborhood $U$ of the identity in $G$ one can find a finite set $F$ in $G$ such that $F U=G=U F$. A standard example of a precompact paratopological group is the unit circle $\mathbb{T}_{\text {Sor }}$ endowed with 
the Sorgenfrey topology which is obtained as the quotient group $\mathbb{S} / \mathbb{Z}$ and where $\mathbb{S}$ is the usual Sorgenfrey line. Notice that the paratopological group $\mathbb{T}_{\text {Sor }}$ is Hausdorff and zero-dimensional (hence completely regular), but it obviously fails to be a topological group.

Similarly, a paratopological group $G$ is $\tau$-narrow, for a given cardinal $\tau \geq \omega$, if for every neighborhood $U$ of the identity in $G$ one can find a subset $F$ of $G$ such that $F U=G=U F$ and $|F| \leq \tau$. The class of $\tau$-narrow paratopological groups is productive, but it is not closed with respect to taking subgroups. In fact, a subgroup of a precompact paratopological group can even fail to be $\omega$-narrow (this follows from [4, Corollary 5]).

Given a Hausdorff space $X$ with topology $\tau$, one defines the semiregularization of $\tau$, denoted by $\tau_{r}$, as a topology on $X$ whose base consists of regular open subsets of $(X, \tau)$ (see $[33,17])$. The topology $\tau_{r}$ is coarser than $\tau$ and the space $X_{r}=\left(X, \tau_{r}\right)$, the semiregularization of $X$, is Hausdorff and semiregular, i.e., the family of regular open sets in $X_{r}$ constitutes a base for $X_{r}$. If $G$ is a Hausdorff paratopological group, then the semiregularization $G_{r}$ of $G$ is a regular paratopological group (see [23, Example 1.9]). This fact permits us to call $G_{r}$ the regularization of $G$.

Let $X=\prod_{i \in I} X_{i}$ be a product of spaces with the usual Tychonoff product topology. For every $i \in I$, denote by $\pi_{i}$ the projection of $X$ onto the factor $X_{i}$. If $U$ is a canonical open set in $X$ (i.e., an element of the canonical base for $X$, see [12, Section 2.3]), we put

$$
c(U)=\left\{i \in I: \pi_{i}(U) \neq X_{i}\right\} .
$$

Clearly $c(U)$ is a finite subset of $I$.

A nonempty subset $S$ of an Abelian group $G$ is called independent if the equality $x_{1}^{n_{1}} \cdot \ldots \cdot x_{k}^{n_{k}}=1_{G}$ with $n_{1}, \ldots, n_{k} \in \mathbb{Z}$ and distinct $x_{1}, \ldots, x_{k} \in S$ implies $x_{1}^{n_{1}}=\cdots=$ $x_{k}^{n_{k}}=1_{G}$. It is clear that a set $S \subseteq G$ consisting of elements of infinite order is independent if and only if the former equality implies that $n_{1}=\cdots=n_{k}=0$. A set $C \subseteq G$ is called a subsemigroup of $G$ if $x \cdot y \in C$ for all $x, y \in C$.

\section{Three examples of feebly compact paratopological groups}

In [1] Sanchis and Alas posed the question whether every 2-pseudocompact Hausdorff topological group $G$ was precompact (or, equivalently, whether $G$ was a topological group). In [25, Problem 1] O. Ravsky asked whether every feebly compact Hausdorff paratopological group with the Baire property was a topological group. He also raised a question equivalent to the one in [1]: Is every 2-pseudocompact Hausdorff paratopological group a topological group? Notice that all 2-pseudocompact paratopological groups have the Baire property by [1, Theorem 2.2]. A consistent counterexample to both questions was presented in [25, Example 2].

In Theorem 1 below we answer all these questions in the negative by constructing in $Z F C$ a 2-pseudocompact, feebly compact Hausdorff paratopological group $G$ that fails to be precompact and, therefore, cannot be a topological group. Then, in Remark 1, we comment on several additional properties of the group $G$ from Theorem 1.

We start with two simple lemmas. 
Lemma 1 Let $\mathbb{T}$ be circle group endowed with the usual topology. One can find a family $\mathcal{S}=\left\{S_{\alpha}: \alpha \in \omega_{1}\right\}$ of infinite pairwise disjoint subsets of $\mathbb{T}$ satisfying the following conditions:

(1) $S_{\alpha}=\left\{t_{\alpha, n}: n \in \omega\right\}$ is dense in $\mathbb{T}$ and consists of elements of infinite order, for each $\alpha \in \omega_{1}$

(2) $S=\bigcup_{\alpha \in \omega_{1}} S_{\alpha}$ is an independent subset of $\mathbb{T}$.

Proof Let $\left\{U_{n}: n \in \omega\right\}$ be a countable neighborhood base for the space $\mathbb{T}$. Suppose that for some $\alpha \in \omega_{1}$, we have defined a family $\left\{S_{v}: v \in \alpha\right\}$ of infinite disjoint subsets of $\mathbb{T}$ such that $S_{v}=\left\{t_{v, n}: n \in \omega\right\}$ is dense in $\mathbb{T}$ for each $v \in \alpha$ and the set $T_{\alpha}=\bigcup_{v \in \alpha} S_{v}$ is independent. We define $S_{\alpha}$ as follows. Since $U_{0}$ is uncountable, there exists an element $t_{\alpha, 0} \in U_{0}$ of infinite order in $\mathbb{T}$ such that $\left\langle t_{\alpha, 0}\right\rangle \cap\left\langle T_{\alpha}\right\rangle=\{1\}$. Suppose that for some $n \in \omega$, we have chosen elements $t_{\alpha, 0} \in U_{0}, \ldots, t_{\alpha, n} \in U_{n}$ of infinite order such that the set $T_{\alpha} \cup S_{\alpha, n}$ is independent in $\mathbb{T}$, where $S_{\alpha, n}=\left\{t_{\alpha, i}: i \leq n\right\}$. Again, since $U_{n+1}$ is uncountable, we can find an element $t_{\alpha, n+1} \in U_{n+1}$ of infinite order such that $\left\langle t_{\alpha, n+1}\right\rangle \cap\left\langle T_{\alpha} \cup S_{\alpha, n}\right\rangle=\{1\}$. Clearly, the set $T_{\alpha} \cup\left\{t_{\alpha, i}: i \leq n+1\right\}$ is independent.

Continuing this way, we obtain a set $S_{\alpha}=\left\{t_{\alpha, n}: n \in \omega\right\}$ which intersects every nonempty open set in $\mathbb{T}$. It also follows from our choice of the elements $t_{\alpha, n}$ that the sets $T_{\alpha}$ and $S_{\alpha}$ are disjoint and $T_{\alpha} \cup S_{\alpha}$ is independent in $\mathbb{T}$. This finishes our construction of the family $\mathcal{S}$ satisfying conditions (1) and (2) of the lemma.

The following result remains valid in the non-Abelian case if one adds an additional assumption about the family $\mathcal{C}$ :

Lemma 2 Let $\Sigma=(\Sigma, \tau)$ be a paratopological Abelian group with identity $e$ and $\mathcal{C}$ a family of subsemigroups of $\Sigma$ closed under finite intersections such that $e \in C$ for each $C \in \mathcal{C}$. Let also $\sigma=\langle\tau, \mathcal{C}\rangle$ be the topology on $\Sigma$ with the base $\{x C \cap U$ : $x \in \Sigma, C \in \mathcal{C}, x \in U \in \tau\}$. Then $G=(\Sigma, \sigma)$ is again a paratopological group. In addition, if the space $\Sigma$ is regular and the family $\mathcal{C}$ satisfies

( $\alpha) C \cap x C$ is dense in $\Sigma$ for all $x \in \Sigma$ and $C \in \mathcal{C}$,

then

(1) $c l_{\Sigma} V=c l_{G}(C \cap V)$, for all $V \in \tau$ and $C \in \mathcal{C}$;

(2) $\sigma_{r}=\tau$, i.e., the regularization of $G$ coincides with $\Sigma$;

(3) $G$ is Hausdorff and if $C \notin \tau$ for some $C \in \mathcal{C}$, then $G$ is not regular.

Proof Let $\mathcal{N}_{e}$ be the family of open neighborhoods of $e$ in $(\Sigma, \tau)$. It is easy to see that the family

$$
\mathcal{B}_{e}=\left\{U \cap C: U \in \mathcal{N}_{e}, C \in \mathcal{C}\right\}
$$

satisfies Pontryagin's four conditions (see [31, Proposition 2.1], hence $\sigma=\langle\tau, \mathcal{C}\rangle$ is a paratopological group topology on $\Sigma$ with $\mathcal{B}_{e}$ being a local base for $G=(\Sigma, \sigma)$ at the identity $e$.

Suppose now that $\Sigma$ is regular and $\mathcal{C}$ satisfies condition $(\alpha)$ of the lemma.

(1) Let $V \in \tau$ and $C \in \mathcal{C}$. Then $c_{G}(C \cap V) \subseteq c l_{G} V \subseteq c l_{\Sigma} V$ since $\sigma$ is finer than $\tau$. Conversely, take any element $x \in c l_{\Sigma} V$ and let $U$ be a neighborhood of $x$ in $G$. Then there exists an open neighborhood $O$ of $x$ in $\Sigma$ and an element $D \in \mathcal{C}$ such that 
$x D \cap O \subseteq U$. To show that $x \in c_{G}(C \cap V)$ we have to verify that $(x D \cap O) \cap(C \cap V) \neq$ $\emptyset$. Clearly, $E=C \cap D$ is in $\mathcal{C}$. Since $x \in c l_{\Sigma} V$ and $V \in \tau$, the set $O \cap V$ is open in $\Sigma$ and nonempty. The density of $x E \cap E$ in $\Sigma$ implies that $\emptyset \neq(x E \cap E) \cap(O \cap V) \subseteq$ $(x D \cap O) \cap(C \cap V)$. This shows that $c l_{\Sigma} V \subseteq c l_{G}(C \cap V)$ and proves (1) of the lemma.

(2) Since $\tau \subseteq \sigma$, the regularity of $\tau$ implies that $\tau \subseteq \sigma_{r}$. To show that $\sigma_{r} \subseteq \tau$ it suffices to note that, by (1), $V \subseteq c l_{\Sigma} V=\operatorname{cl}_{G}(V \cap C)$ for all $V \in \tau$ and $C \in \mathcal{C}$. We conclude, therefore, that $\sigma_{r}=\tau$.

(3) It is clear that $G$ is Hausdorff since the topology $\sigma$ of $G$ finer than the regular (hence Hausdorff) topology $\tau$. If $C \notin \tau$ for some $C \in \mathcal{C}$, then $\sigma$ is strictly finer than $\tau$. Since $\sigma_{r}=\tau$ by (2), the topology $\sigma$ cannot be regular.

Theorem 1 There exists a feebly compact, 2-pseudocompact Hausdorff paratopological group $G$ with the Fréchet-Urysohn property which fails to be a topological group. Furthermore, $G$ has the Baire property, is countably cellular, but it is neither regular nor $\omega$-narrow (nor precompact).

Proof Let $\Pi$ be the compact group $\mathbb{T}^{\omega_{1}}$ with the usual pointwise multiplication and Tychonoff product topology. We will use multiplicative notation for the group operation $\mathbb{T}$, so 1 is the identity element of $\mathbb{T}$.

For every element $x \in \Pi$, let

$$
\operatorname{supp}(x)=\left\{\alpha<\omega_{1}: x(\alpha) \neq 1\right\} .
$$

Then $\Sigma=\{x \in \Pi:|\operatorname{supp}(x)| \leq \omega\}$ is a dense subgroup of $\Pi$. In addition, the group $\Sigma$ has the Fréchet-Urysohn property [3, Corollary 1.6.33].

Let $S=\left\{t_{n}: n \in \omega\right\}$ be a dense independent subset of $\mathbb{T}$ whose elements have infinite order (see Lemma 1). Denote by $K$ the subgroup of $\mathbb{T}$ generated by $S$. Every element $t \in K$ distinct from 1 has a unique representation in the form $t=t_{n_{1}}^{p_{1}} \cdots t_{n_{k}}^{p_{k}}$, where $k$ is a positive integer, $0 \leq n_{1}<\cdots<n_{k}<\omega$, and $p_{1}, \ldots, p_{k}$ are nonzero integers. For such an element $t \in K$, we put

$$
p(t)=\sum_{1 \leq i \leq k} p_{i}
$$

We can now define a subset $C_{0}$ of $\Sigma$ by

$$
C_{0}=\left\{x \in \Sigma:\left(\exists \alpha<\omega_{1}\right)[x(\alpha) \in K \& p(x(\alpha))>0 \&(\forall \beta>\alpha)[x(\beta)=1]]\right\} .
$$

It is clear from the definition that $C=C_{0} \cup\{e\}$ is a subsemigroup of $\Sigma$, i.e., $a \cdot b \in C$ for all $a, b \in C$; here $e$ stands for the identity element of $\Sigma$.

Let us refine the topology $\tau$ of $\Sigma$ inherited from $\Pi$ by declaring the set $C$ open. In other words, a base of the new topology of $\Sigma$, say $\sigma$, consists of the sets $x C \cap U$, where $x \in \Sigma$ and $x \in U \in \tau$. Since $C$ is a subsemigroup of $\Sigma$ and $\sigma$ is finer than the original topology $\tau$ of $\Sigma$, it follows from Lemma 2 that $G=(\Sigma, \sigma)$ is a Hausdorff paratopological group. It is immediate from the definition of $C$ that both $C$ and $C^{-1}$ are dense in $(\Sigma, \tau)$. In fact, we claim that $C$ satisfies condition $(\alpha)$ of Lemma 2, i.e., the set $x C \cap C$ is dense in $(\Sigma, \tau)$ for each $x \in \Sigma$.

Indeed, for every $\alpha \in \omega_{1}$, let $D_{\alpha}$ be the set of all $x \in C_{0}$ such that $\max \operatorname{supp}(x) \geq \alpha$. Clearly, $D_{\alpha}$ is a dense subsemigroup of $(\Sigma, \tau)$ and $D_{\alpha} \subseteq C_{0} \subseteq C$. Take an arbitrary 
element $x \in \Sigma$ and choose $\alpha \in \omega_{1}$ such that $\operatorname{supp}(x) \subseteq \alpha$. It is clear that $x d \in D_{\alpha} \subseteq C$ for each $d \in D_{\alpha}$ and, hence, $x D_{\alpha} \subseteq x C \cap C$. Since the sets $D_{\alpha}$ and $x D_{\alpha}$ are dense in $(\Sigma, \tau)$, so is $x C \cap C$. This proves our claim.

For the further argument we formulate a simple property of the subsemigroups $D_{\alpha}$ 's which is immediate from the definition of the subsemigroups (and which has been implicitly used in the previous paragraph):

Claim 1. If $\alpha<\omega_{1}$ and an element $x \in \Sigma$ satisfies $\operatorname{supp}(x) \subseteq \alpha$, then $x D_{\alpha}=D_{\alpha}$.

(A) It is clear that $C$ is not open in $(\Sigma, \tau)$, so the space $G$ is not regular by (3) of Lemma 2. Since every Hausdorff topological group is a regular space, we see that $G$ fails to be a topological group.

(B) Our first aim is to verify that $G$ has countable tightness (see [12, 1.7.13]). Suppose that $x \in \operatorname{cl}_{G} P$, for some $x \in G$ and $P \subseteq G$. We claim that there exists a countable set $Q \subseteq P$ such that $x \in \operatorname{cl}_{G} Q$. Our argument involves a standard inductive construction.

Take an arbitrary nonempty countable set $A_{0} \subseteq \omega_{1}$ such that $\operatorname{supp}(x) \subseteq A_{0}$. Let us identify every point $y \in \mathbb{T}^{A_{0}}$ with the corresponding point $\bar{y}$ of $\Sigma$ which coincides with $y$ on $A_{0}$ and $\bar{y}(\alpha)=1$ for each $\alpha \in \omega_{1} \backslash A_{0}$. Since the subspace $\mathbb{T}^{A_{0}}$ of $(\Sigma, \tau)$ is first countable, there exists a countable set $Q_{0} \subseteq P$ with the property that $x C \cap U \cap Q_{0} \neq \emptyset$ for every canonical open neighborhood $U$ of $x$ in $\Pi$ such that $c(U) \subseteq A_{0}$.

Suppose that for some $n \in \omega$, we have defined countable sets $A_{n} \subseteq \omega_{1}$ and $Q_{n} \subseteq P$ with the property that $x C \cap U \cap Q_{n} \neq \emptyset$ for every canonical open neighborhood $U$ of $x$ in $\Pi$ such that $c(U) \subseteq A_{n}$. Then we put $A_{n+1}=A_{n} \cup \bigcup_{y \in Q_{n}} \operatorname{supp}(y)$. Since the set $A_{n+1}$ is countable and the space $\mathbb{T}^{A_{n+1}}$ is first countable, there exists a countable set $Q_{n+1} \subseteq P$ with the property that $x C \cap U \cap Q_{n+1} \neq \emptyset$ for every canonical open neighborhood $U$ of $x$ in $\Pi$ such that $c(U) \subseteq A_{n+1}$. This finishes our construction of the sequences $\left\{A_{n}: n \in \omega\right\}$ and $\left\{Q_{n}: n \in \omega\right\}$.

Let $A=\bigcup_{n \in \omega} A_{n}$ and $Q=\bigcup_{n \in \omega} Q_{n}$. Clearly, the sets $A \subseteq \omega_{1}$ and $Q \subseteq P$ are countable. It follows from our construction that $\operatorname{supp}(x) \subseteq A_{0} \subseteq A$ and $\operatorname{supp}(y) \subseteq A$ for each $y \in Q$. It remains to show that $x \in c l_{G} Q$. Take an arbitrary open neighborhood $U$ of $x$ in $G$. There exists a canonical open neighborhood $V$ of $x$ in $\Pi$ such that $x C \cap V \subseteq U$. Then $B=c(V) \cap A$ is a finite subset of $A$. Since the sequence $\left\{A_{n}: n \in\right.$ $\omega\}$ is increasing, we can find $n \in \omega$ such that $B \subseteq A_{n}$. Let $W=\pi_{A}^{-1} \pi_{A}(V)$, where $\pi_{A}: \Pi \rightarrow \mathbb{T}^{A}$ is the projection. Then $W$ is a canonical open neighborhood of $x$ in $\Pi$ and $c(W)=B \subseteq A_{n}$. Hence, by our choice of $Q_{n}$, there exists a point $y \in x C \cap W \cap Q_{n}$. Since $Q_{n} \subseteq Q$, we see that $\operatorname{supp}(y) \subseteq A$. In particular, $y(\alpha)=1$ for each $\alpha \in \omega_{1} \backslash A$. This implies that $y \in x C \cap V$ since the canonical open set $V$ contains the point $x$ and, therefore, $1 \in \pi_{\alpha}(V)$ for each $\alpha \in \omega_{1} \backslash A$ (notice that $\pi_{\alpha}(V)=\pi_{\alpha}(W)$ for each $\alpha \in A$ ). Therefore, $x C \cap V \cap Q \neq \emptyset$ and we conclude that $x \in c l_{G} Q$. This proves that $G$ has countable tightness.

The stronger fact that $G$ has the Fréchet-Urysohn property is now almost immediate. First, every countable subspace of $(\Sigma, \tau)$ is first countable (see [3, Proposition 1.6.31]). Hence, by the definition of the topology $\sigma$ on $\Sigma$, the same holds for countable subspaces of the space $G=(\Sigma, \sigma)$. If $x \in c l_{G} P$ for some $x \in G$ and $P \subseteq G$, we already know that there exists a countable set $Q \subseteq P$ such that $x \in c l_{G} Q$. Since the subspace $\{x\} \cup Q$ of $G$ is first countable, it has a countable local base at the point 
$x$. Therefore, there exists a sequence from $Q$ converging to $x$, i.e., $G$ has the FréchetUrysohn property.

(C) To show that $G$ is 2-pseudocompact we need to establish the following:

Claim 2. For every nonempty open set $V$ in $\Sigma=(\Sigma, \tau)$, there exists an ordinal $\alpha_{V} \in$ $\omega_{1}$ such that

$$
D_{\alpha}^{-1} \cap c l_{\Sigma} V \subseteq c l_{G}\left(D_{\alpha}^{-1} \cap V\right)
$$

whenever $\alpha_{V} \leq \alpha<\omega_{1}$.

Proof of Claim 2. Let $V$ be a nonempty open set in $(\Sigma, \tau)$. Take an open set $\widetilde{V}$ in $\Pi$ such that $\widetilde{V} \cap \Sigma=V$. Since $\Pi$ has countable cellularity, there exists a countable family $\gamma$ of canonical open sets in $\Pi$ such that $\bigcup \gamma$ is a dense subset of $\widetilde{V}$. Clearly we can assume that each $U \in \gamma$ is a proper subset of $\mathbb{T}^{\omega_{1}}$, so $c(U) \neq \emptyset$ for each $U \in \gamma$. Then $A=\bigcup_{U \in \gamma} c(U)$ is a countable subset of $\omega_{1}$ and we can find an ordinal $\alpha_{V} \in \omega_{1}$ such that $A \subseteq \alpha_{V}$. Let us show that $\alpha_{V}$ is as required.

Take an ordinal $\alpha$ with $\alpha_{V} \leq \alpha<\omega_{1}$ and an arbitrary point $x \in D_{\alpha}^{-1} \cap c l_{\Sigma} V$ and let $O \cap x C$ be a basic open neighborhood of $x$ in $G$, where $O$ is a canonical open neighborhood of $x$ in $\Pi$. We have to verify that $(O \cap x C) \cap\left(D_{\alpha}^{-1} \cap V\right) \neq \emptyset$ or, equivalently, that $(O \cap x C) \cap\left(D_{\alpha}^{-1} \cap U\right) \neq \emptyset$ for some $U \in \gamma$.

Since $x \in c l_{\Sigma} V$ and $x \in O \cap \Sigma \in \tau$, it follows from our choice of the family $\gamma$ that $O \cap U \neq \emptyset$ for some $U \in \gamma$. Let $O=\prod_{\beta \in \omega_{1}} O_{\beta}$ and $U=\prod_{\beta \in \omega_{1}} U_{\beta}$. Then $U_{\beta}=\mathbb{T}$ for each $\beta \geq \alpha$ since $c(U) \subseteq \alpha_{V} \subseteq \alpha$. Put $\delta=\max c(U)$ and note that $\delta<\alpha$. We define elements $c, d \in C$ as follows. First, $O_{\delta} \cap U_{\delta}$ is a nonempty open subset of $\mathbb{T}$, so we use the density of $S=\left\{t_{n}: n \in \omega\right\}$ in $\mathbb{T}$ to take $n \in \omega$ such that $t_{n} \in\left(O_{\delta} \cap\right.$ $\left.U_{\delta}\right) \cdot x(\delta)^{-1}$. Let $c(\delta)=t_{n}$ and $c(\beta)=1$ for each $\beta>\delta$. Hence, independently of the choice of the values $c(\beta)$ for $\beta<\delta$, we will have that $c \in D_{\delta} \subseteq C$. Let also $d(\delta)=c(\delta)^{-1} \cdot x(\delta)^{-1}$. Then $d(\delta)^{-1}=c(\delta) \cdot x(\delta) \in U_{\delta} \cap O_{\delta}$. We put $d(\beta)=x(\beta)^{-1}$ for each $\beta>\delta$. Then $d(\beta)^{-1}=x(\beta) \in O_{\beta}$. Finally, given an ordinal $\beta<\delta$, pick an element $d(\beta) \in O_{\beta}^{-1} \cap U_{\beta}^{-1}$ and put $c(\beta)=d(\beta)^{-1} \cdot x(\beta)^{-1}$. It is immediate from our definitions that $x c=d^{-1}$. Since $x^{-1} \in D_{\alpha}$ and $d(\beta)=x(\beta)^{-1}$ for each $\beta \geq \alpha>\delta$, we see that $d \in D_{\alpha}$. Our definition of the element $d$ and the facts that $U$ and $O$ are canonical open sets in $\Pi$ and $\max c(U)=\delta$ imply that $d^{-1} \in O \cap U$. Therefore,

$$
x c=d^{-1} \in(O \cap U) \cap\left(x C \cap D_{\alpha}^{-1}\right)=(O \cap x C) \cap\left(D_{\alpha}^{-1} \cap U\right) \neq \emptyset .
$$

This implies that $x \in c l_{G}\left(D_{\alpha}^{-1} \cap V\right)$ and finishes the proof of Claim 2 .

Let us turn back to the proof of the 2-pseudocompactness of $G$. Suppose that $\left\{U_{n}: n \in \omega\right\}$ is a decreasing sequence of nonempty open sets in $G$. For every $n \in \omega$, choose $x_{n} \in U_{n}$ and an open neighborhood $V_{n}$ of $x_{n}$ in $\Pi$ such that $x_{n} C \cap V_{n} \subseteq U_{n}$. Put $O_{n}=\bigcup_{k \geq n}\left(x_{k} C \cap V_{k}\right)$. It is clear that $O_{n}$ is open in $G$ and $O_{n} \subseteq U_{n}$ for each $n \in \omega$. Hence it suffices to verify that the set $\bigcap_{n \in \omega} c l_{G} O_{n}^{-1}$ is nonempty.

For every $n \in \omega$, put $W_{n}=\bigcup_{k>n} V_{k}$. Then $\left\{W_{n}: n \in \omega\right\}$ is a decreasing sequence of nonempty open sets in $\Pi$. Applying Claim 2 we find, for every $n \in \omega$, an ordinal $\alpha_{n} \in \omega_{1}$ such that $D_{\alpha}^{-1} \cap c l_{\Sigma} W_{n}^{-1} \subseteq \operatorname{cl}_{G}\left(D_{\alpha}^{-1} \cap W_{n}^{-1}\right)$ for each $\alpha$ with $\alpha_{n} \leq \alpha<\omega_{1}$. 
Take a countable ordinal $\beta$ such that $\alpha_{n} \leq \beta$ and $\operatorname{supp}\left(x_{n}\right) \subseteq \beta$ for each $n \in \omega$. By Claims 1 and 2, we have that

$$
\begin{aligned}
\bigcap_{n \in \omega} c l_{G} O_{n}^{-1} & =\bigcap_{n \in \omega} c l_{G}\left(\bigcup_{k \geq n} x_{k}^{-1} C^{-1} \cap V_{k}^{-1}\right) \supseteq \bigcap_{n \in \omega} c l_{G}\left(\bigcup_{k \geq n} x_{k}^{-1} D_{\beta}^{-1} \cap V_{k}^{-1}\right) \\
& =\bigcap_{n \in \omega} c l_{G}\left(\bigcup_{k \geq n} D_{\beta}^{-1} \cap V_{k}^{-1}\right)=\bigcap_{n \in \omega} c l_{G}\left(D_{\beta}^{-1} \cap W_{n}^{-1}\right) \\
& \supseteq \bigcap_{n \in \omega}\left(D_{\beta}^{-1} \cap c l_{\Sigma} W_{n}^{-1}\right)=D_{\beta}^{-1} \cap \bigcap_{n \in \omega} c l_{\Sigma} W_{n}^{-1} .
\end{aligned}
$$

Since the space $\Pi$ is compact, the intersection $\bigcap_{n \in \omega} c l_{\Pi} W_{n}^{-1}$ is nonempty. The sets $W_{n}$ 's being open in $\Pi$, each $c l_{\Pi} W_{n}^{-1}$ is a $G_{\delta}$-set in $\Pi$ according to [3, Corollary 1.6.19]. Hence $\bigcap_{n \in \omega} c l_{\Pi} W_{n}^{-1}$ is also a $G_{\delta}$-set in $\Pi$. Since the sets $D_{\beta}$ and $D_{\beta}^{-1}$ meet every nonempty $G_{\delta}$-set in $\Pi$, we conclude that $D_{\beta}^{-1} \cap \bigcap_{n \in \omega} c l_{\Sigma} W_{n}^{-1} \neq \emptyset$. Therefore, the intersection $\bigcap_{n \in \omega} c l_{G} O_{n}^{-1}$ is nonempty and $G$ is 2-pseudocompact.

(D) We already know that the regularization of $G=(\Sigma, \sigma)$ coincides with the countably compact (hence pseudocompact) topological group $(\Sigma, \tau)$. Since a paratopological group is feebly compact iff so is its regularization (see [25, Lemma 13]), we conclude that $G$ is feebly compact.

Notice that every 2-pseudocompact paratopological group has the Baire property (see [1, Theorem 2.2] or [25, Lemma 4]) and the cellularity of every feebly compact paratopological group is countable [27].

Finally, we show that $G$ cannot be covered by countably many translates of the open set $C$, which implies that $G$ is neither $\omega$-narrow nor precompact. Take any countable set $F \subseteq G$. Since $F$ is a subset of $\Sigma$, there exists $\alpha^{*} \in \omega_{1}$ such that $x(\alpha)=1$ for all $x \in F$ and $\alpha \geq \alpha^{*}$. Let $y$ be an element of $G$ such that $y\left(\alpha^{*}\right) \notin K$ and $y(\alpha)=1$ for each $\alpha>\alpha^{*}$. It is clear that $y \in G \backslash F C$, i.e., $G$ is not $\omega$-narrow.

Remark 1 a) The topology $\tau$ of the group $\Sigma$ inherited from $\Pi$ is the finest topological group topology on $\Sigma$ coarser than the topology $\sigma$ on $\Sigma$ defined in Theorem 1 . In other words, using the terminology of [4], one can say that the group reflection of the paratopological group $G$ is topologically isomorphic to the subgroup $(\Sigma, \tau)$ of $\Pi$.

Indeed, since the paratopological group $G=(\Sigma, \sigma)$ is commutative, a base of open neighborhoods of the group reflection of $G$ at the identity $e$ consists of the sets $W \cdot W^{-1}$, where $W$ runs over the family of open neighborhoods of $e$ in $G$ (this follows from [5, Theorem 1]). Every neighborhood of $e$ in $G$ contains a set $W$ of the form $V \cap C$, where $V$ is a canonical open neighborhood of $e$ in $\Pi$. A simple calculation shows that $V \cap \Sigma \subseteq W W^{-1}$, whence it follows that the group reflection of $G$ is topologically isomorphic to $(\Sigma, \tau)$.

b) The group $G$ is not precompact, but it is topologically isomorphic to a subgroup of a precompact Hausdorff paratopological group. Indeed, the identity mapping of $G=(\Sigma, \sigma)$ onto $(\Sigma, \tau)$ is a continuous isomorphism of $G$ onto a precompact topological group. Therefore, according to [4, Corollary 5], $G$ admits a topological embedding as a subgroup into a precompact Hausdorff paratopological group. 
c) A subset $B$ of a space $Y$ is said to be (feebly) bounded in $X$ if every locally finite family of open sets in $X$ meeting $B$ is finite. According to [16, Theorem 1.2], if $A$ is a bounded subset of a topological group $X$ and $B$ is a (feebly) bounded subset of a space $Y$, then the product $A \times B$ is (feebly) bounded in $X \times Y$. We claim that the paratopological group $G$ has a slightly weaker property: For every space $Y, G \times B$ is (feebly) bounded in $X \times Y$, for every (feebly) bounded subset $B$ of $Y$. In particular, $G \times$ $Y$ is feebly compact, for every feebly compact space $Y$. In other words, the space $G$ belongs to the natural analog of Frolík's class $\mathcal{P}$ obtained by omitting any separation restrictions on the factors (see [13]). According to [35, Theorem 1.2] and [6, Proposition 1], both facts will follow if we show that every infinite disjoint family $\gamma$ of nonempty open sets in $G$ contains a disjoint subfamily $\left\{U_{n}: n \in \omega\right\}$ such that the set

$$
Q=\bigcap_{F \in \mathcal{F}}^{\infty} c l_{G}\left(\bigcup_{n \in F} U_{n}\right)
$$

is nonempty for every filter $\mathcal{F}$ of infinite subsets of $\omega$. In fact, we will show that the set $Q$ in (1) is nonempty for every sequence $\left\{U_{n}: n \in \omega\right\}$ of nonempty open sets in $G$ and every filter $\mathcal{F}$ on $\omega$. We can assume without loss of generality that each $U_{n}$ has the form $x_{n} C \cap V_{n}$, where $V_{n}$ is a canonical open set in $\Pi$ with $x_{n} \in V_{n}$.

First we note that if $X$ is a space and $X_{r}$ is semiregularization of $X$, then $c l_{X} U=$ $c l_{X_{Y}} U$ for each open subset $U$ of $X$. This follows immediately from the fact that $V=$ $X \backslash c l_{X} U$ is a regular open set in $X$ and, hence, $V$ is open in $X_{r}$. Therefore,

$$
c l_{G}\left(\bigcup_{n \in F} U_{n}\right)=c l_{\Sigma}\left(\bigcup_{n \in F} U_{n}\right)=c l_{\Sigma}\left(\bigcup_{n \in F}\left(\Sigma \cap V_{n}\right)\right)=c l_{\Sigma}\left(\Sigma \cap \bigcup_{n \in F} V_{n}\right)
$$

for each $F \in \mathcal{F}$, since $U_{n}=x_{n} C \cap V_{n}$ is dense in the open subspace $\Sigma \cap V_{n}$ of $\Sigma$ for each $n \in \omega$.

Since every $V_{n}$ depends on finitely many coordinates, there exists a countable set $A \subset \omega_{1}$ such that $c\left(V_{n}\right) \subseteq A$ and, hence, $V_{n}=\pi_{A}^{-1} \pi_{A}\left(V_{n}\right)$ for each $n \in \omega$. Put

$$
R=\bigcap_{F \in \mathcal{F}} c l_{\Pi}\left(\bigcup_{n \in F} V_{n}\right) .
$$

It follows from the compactness of $\Pi$ that the set $R$ is not empty. In addition, since $c\left(V_{n}\right) \subseteq A$ for each $n \in \omega$ and the projection $\pi_{A}: \mathbb{T}^{\omega_{1}} \rightarrow \mathbb{T}^{A}$ is open, we conclude that $R=\pi_{A}^{-1} \pi_{A}(R)$. Take any point $x \in R \cap \Sigma$. It follows from (2) that $x \in c l_{G}\left(\bigcup_{n \in F} U_{n}\right)$ for each $F \in \mathcal{F}$, i.e., the set $Q$ in (1) is not empty. This finishes the proof that the product $G \times Y$ is feebly compact for every feebly compact space $Y$.

d) It is a well-known fact that every pseudocompact topological group $G$ is precompact in its finest uniformity (which coincides with any of the natural uniformities on $G$, see [10] or [34, Corollary 2.13]). The situation is quite different for paratopological groups. Indeed, since precompact 2-pseudocompact paratopological groups are topological groups, Theorem 1 provides an example of a feebly compact, 2pseudocompact Hausdorff paratopological group whose finest quasi-uniformity fails to be precompact (compare with [30, Lemma 3.3]). 
It is easy to verify that every infinite pseudocompact topological group contains a countable non-closed subset (see [3, Corollary 1.4.24]). Since pseudocompact topological groups are precompact by [10, Theorem 1.1], the former fact is an immediate corollary to Protasov's theorem in [22]: Every infinite precompact topological group contains a countable discrete subset that accumulates at the identity of the group. In the next theorem we show that feebly compact Hausdorff paratopological groups can fail to have this property.

Theorem 2 There exists an infinite feebly compact Hausdorff paratopological group $G$ with the Baire property such that all countable subsets of $G$ are closed. In addition, $G$ contains a nonempty open subsemigroup $C$ such that the set $C^{-1}$ is closed and discrete in $G$, so $G$ is not 2-pseudocompact.

Proof As in the proof of Theorem 1, let $\Sigma$ be the $\Sigma$-product of $\omega_{1}$ copies of the circle group $\mathbb{T}$ endowed with the topology $\tau$ inherited from the product group $\Pi=\mathbb{T}^{\omega_{1}}$. Then $\Sigma$ is a countably compact (hence pseudocompact) topological group. Let us refine the topology of $\Sigma$ by declaring a family $\left\{C_{\alpha}: \alpha \in \omega_{1}\right\}$ of dense subsemigroups of $\Sigma$ to be open in the new topology. In what follows we use notation introduced in the proof of Theorem 1. In particular, we take a dense independent subset $S=\left\{t_{n}: n \in \omega\right\}$ of $\mathbb{T}$ consisting of elements of infinite order and denote by $K$ the subgroup of $\mathbb{T}$ generated by $S$.

For a countable ordinal $\alpha$, let

$$
D_{\alpha}=\{x \in \Sigma:(\exists \beta \geq \alpha)[x(\beta) \in K \& p(x(\beta))>0 \&(\forall \gamma>\beta)[x(\gamma)=1]]\}
$$

and

$$
C_{\alpha}=D_{\alpha} \cup\{e\},
$$

where $e$ is the neutral element of $\Sigma$. It is clear that each $C_{\alpha}$ is a dense subsemigroup of $\Sigma$ and that $C_{\nu} \subseteq C_{\mu}$ whenever $\mu<v<\omega_{1}$.

Since the family $\mathrm{C}=\left\{C_{\alpha}: \alpha \in \omega_{1}\right\}$ is closed under finite intersections, Lemma 2 implies that $\sigma=\langle\tau, \mathcal{C}\rangle$ is a paratopological group topology on $\Sigma$. Let $G=(\Sigma, \sigma)$. Since $\tau \subseteq \sigma$, the paratopological group $G=(\Sigma, \sigma)$ is Hausdorff.

Let us verify that the family $\mathcal{C}$ satisfies condition $(\alpha)$ of Lemma 2 . Take arbitrary $x \in \Sigma$ and $\alpha \in \omega_{1}$. Choose $\beta \in \omega_{1}$ such that $\alpha \leq \beta$ and $\operatorname{supp}(x) \subseteq \beta$ and take any element $d \in D_{\beta}$. It is clear that $x d \in C_{\alpha}$ and, hence, $x D_{\beta} \subseteq x C_{\beta} \cap C_{\alpha} \subseteq x C_{\alpha} \cap C_{\alpha}$. Since the sets $C_{\beta}$ and $D_{\beta}=C_{\beta} \backslash\{e\}$ are dense in $(\Sigma, \tau)$, so are $x D_{\beta}$ and $x C_{\alpha} \cap C_{\alpha}$. This implies $(\alpha)$. Hence, according to Lemma $2, \sigma_{r}=\tau$.

(A) By [25, Lemma 13], the space $G=(\Sigma, \sigma)$ is feebly compact if and only if so is $G_{r}=(\Sigma, \tau)$. Since the space $G_{r}$ is countably compact (see [3, Corollary 1.6.34]), we deduce that $G$ is feebly compact.

(B) To show that $G$ has the Baire property, we first note that for arbitrary sequences $\left\{\alpha_{n}: n \in \omega\right\} \subseteq \omega_{1}$ and $\left\{x_{n}: n \in \omega\right\} \subseteq G$, there exists an ordinal $\alpha \in \omega_{1}$ such that $D_{\alpha} \subseteq \bigcap_{n \in \omega} x_{n} C_{\alpha_{n}}$. Indeed, take $\alpha \in \omega_{1}$ such that $\alpha_{n} \leq \alpha$ and $\operatorname{supp}\left(x_{n}\right) \subseteq \alpha$ for each $n \in \omega$. It follows from our choice of $\alpha$ and the definition of $D_{\alpha}$ that $x_{n} D_{\alpha}=D_{\alpha}$ for each $n \in \omega$. Therefore, $D_{\alpha}=\bigcap_{n \in \omega} x_{n} D_{\alpha} \subseteq \bigcap_{n \in \omega} x_{n} C_{\alpha_{n}}$.

Suppose for a contradiction that $G$ fails to have the Baire property. Then we can find a sequence $\left\{W_{n}: n \in \omega\right\}$ of open dense sets in $G$ and a nonempty open set $O$ in $G$ 
such that $O \cap \bigcap_{n \in \omega} W_{n}=\emptyset$. Choose a point $x_{0} \in O \cap W_{0}$ and take an ordinal $\alpha_{0} \in \omega_{1}$ and an open set $U_{0}$ in $(\Sigma, \tau)$ containing $x_{0}$ such that $V_{0}=x_{0} C_{\alpha_{0}} \cap U_{0} \subseteq O \cap W_{0}$. Suppose that we have defined a basic open set $V_{n}=x_{n} C_{\alpha_{n}} \cap U_{n}$ in $G$ such that $x_{n} \in$ $V_{n} \subseteq W_{n}$. Then we choose a point $x_{n+1} \in W_{n+1} \cap U_{n}$, a countable ordinal $\alpha_{n+1}$ and an open neighborhood $U_{n+1}$ of $x_{n+1}$ in $(\Sigma, \tau)$ such that $V_{n+1}=x_{n+1} C_{\alpha_{n+1}} \cap U_{n+1} \subseteq W_{n+1}$ and $c l_{\Sigma} U_{n+1} \subseteq U_{n}$. This finishes our construction of the sequences $\left\{\alpha_{n}: n \in \omega\right\} \subseteq$ $\omega_{1},\left\{x_{n}: n \in \omega\right\} \subseteq G$, and $\left\{U_{n}: n \in \omega\right\} \subseteq \tau$. We have just proved that there exists an ordinal $\alpha \in \omega_{1}$ such that $D_{\alpha} \subseteq \bigcap_{n \in \omega} x_{n} C_{\alpha_{n}}$. Since the space $\{\Sigma, \tau\}$ is countably compact, the intersection $P=\bigcap_{n \in \omega} U_{n}=\bigcap_{n \in \omega} c l_{\Sigma} U_{n}$ is a nonempty $G_{\delta}$-set in $(\Sigma, \tau)$. Therefore, $D_{\alpha} \cap P \neq \emptyset$ since $D_{\alpha}$ meets all nonempty $G_{\delta}$-sets in $(\Sigma, \tau)$. This implies that the intersection $\bigcap_{n \in \omega} V_{n}$ is nonempty. However, $\bigcap_{n \in \omega} V_{n} \subseteq O \cap \bigcap_{n \in \omega} W_{n}=\emptyset$, which is a contradiction. Thus $G$ has the Baire property.

(C) Let us show that all countable subsets of $G$ are closed. Take an arbitrary countable set $A \subseteq G \backslash\{e\}$. It suffices to show that $e \notin c l_{G}(A)$. Since $|A| \leq \omega$, we can find an ordinal $\alpha \in \omega_{1}$ such that $\operatorname{supp}(x) \subseteq \alpha$ for each $x \in A$. It follows from the definition of $C_{\alpha}$ that $C_{\alpha} \cap A=\emptyset$ and since $e \in C_{\alpha} \in \sigma$, we conclude that $e \notin c l_{G}(A)$.

(D) Here we verify that $C^{-1}$ is closed and discrete in $G$, where $C=C_{0}$. If $x \in$ $G \backslash C^{-1}$, then $x C \cap C^{-1}=\emptyset$-otherwise $x \in C^{-1} \cdot C^{-1}=C^{-1}$. So $C^{-1}$ is closed in $G$. Further, take any $x \in C^{-1}$ and choose $\alpha \in \omega_{1}$ such that $\operatorname{supp}(x) \subseteq \alpha$. Then $x C_{\alpha} \cap$ $C^{-1}=\{x\}$. Indeed, it follows from $e \in C_{\alpha}$ that $x \in x C_{\alpha} \cap C^{-1}$. It is also clear from the choice of $\alpha$ that $x d \in D_{\alpha}$ for each $d \in D_{\alpha}$, while the sets $D_{\alpha}$ and $C^{-1}$ are disjoint. Hence $x D_{\alpha} \cap C^{-1}=\emptyset$ and $x C_{\alpha} \cap C^{-1}=\{x\}$. Therefore $C^{-1}$ is discrete.

(E) It remains to show that $G$ is not 2-pseudocompact. Take any decreasing sequence $\left\{U_{n}: n \in \omega\right\}$ of nonempty open sets in $(\Sigma, \tau)$ with empty intersection. Since $C=C_{0}$ is dense in $(\Sigma, \tau)$, each set $V_{n}=U_{n} \cap C$ is nonempty and open in $G$. However, the intersection

$$
\bigcap_{n \in \omega} c l_{G} V_{n}^{-1}=\bigcap_{n \in \omega} c l_{G}\left(C^{-1} \cap U_{n}^{-1}\right)=\bigcap_{n \in \omega}\left(C^{-1} \cap U_{n}^{-1}\right)=C^{-1} \cap\left(\bigcap_{n \in \omega} U_{n}\right)^{-1}
$$

is empty and the group $G$ fails to be 2-pseudocompact. This finishes the proof of the theorem.

The group $G$ in Theorem 2 is not 2-pseudocompact since it contains a nonempty open subsemigroup $C$ such that $C^{-1}$ is closed and discrete in $G$. Modifying the construction in Theorem 1, we present below an example of a feebly compact, 2pseudocompact Hausdorff paratopological group $H$ such that all countable subsets of $H$ are closed.

Theorem 3 There exists an infinite feebly compact, 2-pseudocompact Hausdorff paratopological group $H$ in which all countable subsets are closed.

Proof We keep notation of Theorem 1 and denote by $\Sigma$ the $\Sigma$-product of $\omega_{1}$ copies of the circle group $\mathbb{T}$ endowed with topology $\tau$ inherited from $\mathbb{T}^{\omega_{1}}$.

Let $\mathcal{S}=\left\{S_{\alpha}: \alpha \in \omega_{1}\right\}$ be a family of infinite disjoint subset of $\mathbb{T}$ satisfying conditions (1) and (2) of Lemma 1 . Then $S=\bigcup_{\alpha \in \omega_{1}} S_{\alpha}$ is an independent subset of $\mathbb{T}$ consisting of elements of infinite order. Every element $x \in\langle S\rangle$ distinct from the identity 
has a unique representation in the form $x=t_{1}^{p_{1}} \cdots t_{n}^{p_{n}}$, where $t_{1}, \ldots, t_{n}$ are pairwise distinct elements of $S$ and $p_{1}, \ldots, p_{n}$ are nonzero integers. We put $p(x)=\sum_{i=1}^{n} p_{i}$. For every $\alpha \in \omega_{1}$, denote by $K_{\alpha}$ the subgroup of $\mathbb{T}$ generated by the set $\bigcup\left\{S_{\beta}: \alpha \leq \beta<\omega_{1}\right\}$. Notice that $K_{\beta} \subseteq K_{\alpha}$ if $\alpha<\beta<\omega_{1}$. We define subsemigroups $E_{\alpha}$ and $F_{\alpha}$ of $\Sigma$, for each $\alpha \in \omega_{1}$, by letting

$$
E_{\alpha}=\left\{x \in \Sigma:\left(\exists \beta \in \omega_{1}\right)\left[x(\beta) \in K_{\alpha} \& p(x(\beta))>0 \&(\forall \gamma>\beta)[x(\gamma)=1]\right]\right\}
$$

and

$$
F_{\alpha}=E_{\alpha} \cup\{e\},
$$

where $e$ is the identity of $\Sigma$. Evidently, $F_{\beta} \subseteq F_{\alpha}$ whenever $\alpha<\beta<\omega_{1}$, so the family $\mathcal{F}=\left\{F_{\alpha}: \alpha \in \omega_{1}\right\}$ is closed under finite intersections.

Let $\sigma=\langle\tau, \mathcal{F}\rangle$ be the topology on $\Sigma$ with the base

$$
\left\{x F_{\alpha} \cap U: x \in \Sigma, \alpha \in \omega_{1}, x \in U \in \tau\right\} .
$$

It follows from Lemma 2 that $H=(\Sigma, \sigma)$ is a Hausdorff paratopological group. We leave to the reader a routine verification of the fact that $\mathcal{F}$ satisfies condition $(\alpha)$ of Lemma 2, i.e., $F_{\alpha} \cap x F_{\alpha}$ is dense in $\Sigma$ for all $x \in \Sigma$ and $\alpha \in \omega_{1}$. Therefore, the regularization of $H$ coincides with $\Sigma=(\Sigma, \tau)$ and the space $H$ is feebly compact since $\Sigma$ is countably compact.

To show that $H$ is 2-pseudocompact, is suffices to note that each $S_{\alpha} \subseteq K_{\alpha}$ is a dense independent subset of $\mathbb{T}$ and apply the argument in the part (C) of the proof of Theorem 1. In particular, one can show without substantial changes that for every nonempty open subset $V$ of $(\Sigma, \tau)$, there exists a countable ordinal $\alpha_{V}$ such that the inclusion $L_{\beta}^{-1} \cap c l_{\Sigma} V \subset c l_{H}\left(L_{\beta}^{-1} \cap V\right)$ is valid for every ordinal $\beta$ satisfying $\alpha_{V} \leq \beta<$ $\omega_{1}$, where $L_{\beta}=\left\{x \in E_{\beta}: \max \operatorname{supp}(x) \geq \beta\right\}$.

Finally, we verify that all countable subsets of $H$ are closed. To this end, it suffices to show that the identity $e$ of $H$ is not in the closure of any countable set $A \subseteq H \backslash\{e\}$. Let $B=F_{0} \cap A$. For every $x \in B$, put $\beta_{x}=\max \operatorname{supp}(x)$ and $t_{x}=x\left(\beta_{x}\right)$. Since the sets $B$ and $T=\left\{t_{x}: x \in B\right\}$ are countable, there exists an ordinal $\alpha \in \omega_{1}$ such that $T$ is contained in the subgroup of $\mathbb{T}$ generated by the set $\left\{t_{v}: v<\alpha\right\}$. Hence $T \cap K_{\alpha}=\emptyset$ and, consequently, $F_{\alpha} \cap B=\emptyset$. It now follows from $F_{\alpha} \subseteq F_{0}$ that $F_{\alpha} \cap A=F_{\alpha} \cap\left(F_{0} \cap\right.$ $A)=F_{\alpha} \cap B=\emptyset$. Since $F_{\alpha}$ is an open neighborhood of $e$ in $H$, we conclude that $e \notin c l_{H} A$. This completes the proof of the theorem.

Remark 2 The paratopological groups $G$ and $H$ constructed in Theorems 2 and 3, respectively, are connected and locally connected. Let us verify the (local) connectedness of $H$, the argument in the case of $G$ is the same. Clearly, the semiregularization $X_{r}$ of an arbitrary space $X$ is connected if and only if $X$ is connected. Since the subspace $(\Sigma, \tau)=H_{r}$ of $\mathbb{T}^{\omega_{1}}$ is connected, so is $H$. As for the local connectedness of $H$, one applies a similar argument along with the fact that the semiregularization $O_{r}$ of an open subspace of a space $X$ is a subspace of the semiregularization $X_{r}$ of $X$.

The groups constructed in Theorems 2 and 3 are not precompact nor $\omega$-narrow (notice that feebly compact precompact paratopological groups are topological groups according to [25, Proposition 2]). This is why we raise the following question: 
Problem 1 Does there exist an infinite precompact Hausdorff paratopological group all countable subsets of which are closed?

We also repeat the last item of Problem 2 from [25]:

Problem 2 Is every 2-pseudocompact regular paratopological group a topological group?

It is known that the answer to Problem 2 is "yes" if 2-pseudocompactness is strengthened to $C$-compactness (see [30, Proposition 3.4]). Below we explain the terminology and extend the latter fact to locally $C$-compact paratopological groups.

A space $X$ which carries two (arbitrary) topologies $\tau$ and $\gamma$ is called a bitopological space and denoted by $(X, \tau, \gamma)$. Notice that a paratopological group $(G, \tau)$ can be regarded as a bitopological space by considering the topologies $\tau$ and $\tau^{-1}$ on $G$. Given a bitopological space $(X, \tau, \gamma)$, a function $f:(X, \tau, \gamma) \rightarrow(\mathbb{R}, u, l)$ is said to be bicontinuous if both $f:(X, \tau) \rightarrow(\mathbb{R}, u)$ and $f:(X, \gamma) \rightarrow(\mathbb{R}, l)$ are continuous functions (here $u$ and $l$ stand for the upper and lower topology on $\mathbb{R}$, respectively).

The notion of 2-pseudocompactness is related to the study of the concept of boundedness in the realm of bitopological spaces. Indeed, 2-pseudocompact $T_{0}$ paratopological groups $(G, \tau)$ can be characterized by the property that every bicontinuous function on $\left(G, \tau, \tau^{-1}\right)$ is bounded (see [14, Corollary 1.5] for a more general result). The situation becomes rather clearer if we move on to another close notion of boundedness, the so called $C$-compactness. A bitopological space is called $C$ compact if $(f \vee g)(X)$ is a compact subset of $\mathbb{R}=(\mathbb{R}, u \vee l)$ whenever $f:(X, \tau, \gamma) \rightarrow$ $(\mathbb{R}, u, l)$ and $g:(X, \gamma, \tau) \rightarrow(\mathbb{R}, u, l)$ are bicontinuous functions. Here $f \vee g$ stands for $\max \{f, g\}$.

By [30, Proposition 3.4], every $C$-compact paratopological group is a pseudocompact topological group. The following result, related to Problem 2, is a local version of this fact; its proof makes use of the theory of quasi-uniformizable topological spaces. We recall that a quasi-uniform space $(X, \mathcal{U})$ is called precompact if for every $U \in \mathcal{U}$ there is a finite set $F$ such that $X=\bigcup_{x \in F} U(x)$, where $U(x)=\{y \in X:(x, y) \in U\}$. The quasi-uniformity $\mathcal{U}$ is said to be hereditarily precompact if the restriction $\mathcal{U} \uparrow$ $(Y \times Y)$ is a precompact quasi-uniformity for every subset $Y \subset X$. A group is called locally $C$-compact if its identity has a $C$-compact neighborhood.

In the theorem below 'regular' does not include either $T_{0}$ or $T_{1}$ separation axiom. The reader whose preference in interpreting regularity follows Engelking's book [12] can skip the major part of the following argument where we pass from a given paratopological group $G$ to a quotient group $G / K$.

Theorem 4 Every locally C-compact regular paratopological group $G$ is a locally pseudocompact topological group.

Proof Let $e$ be the neutral element of $G$ and $C$ the closure of $\{e\}$ in $G$. Notice that $C \subseteq V^{-1}$ for every neighborhood $V$ of the neutral element $e$ of $G$. It is easy to see that $K=C \cap C^{-1}$ is a normal (perhaps nonclosed) subgroup of $G$. Indeed, both $C$ and $C^{-1}$ are subsemigroups of $G$ since so is $\{e\}$ (see [3, Proposition 1.4.10]). It is also clear that $K^{-1}=K$, so $K$ is a subgroup of $G$. It follows from the definition of $C$ and 
the continuity of inner automorphisms in $G$ that $x^{-1} C x \subseteq C$ for each $x \in G$, i.e., the subsemigroup $C$ is invariant in $G$. Therefore $C^{-1}$ is also invariant in $G$ and so is the subgroup $K=C \cap C^{-1}$ of $G$.

We claim that $U K=U$ for every open set $U \subseteq G$. If not, we can find elements $u \in U$ and $k \in K$ such that $u k \notin U$. Then $V=u^{-1} U$ is an open neighborhood of the neutral element $e$ in $G$ which does not contain $k$, thus contradicting the fact that $k \in K \subseteq V$.

Let $\pi: G \rightarrow G / K$ be the quotient homomorphism of paratopological groups. Clearly $\pi$ is an open mapping. The above observation implies that $U=\pi^{-1} \pi(U)$ for every open set $U \subseteq G$. Hence the quotient paratopological group $G / K$ is a regular space.

We claim that $G / K$ is a $T_{0}$-space. Indeed, by the homogeneity argument, it suffices to verify that for every $\bar{x} \in G / K$ distinct from the neutral element $\bar{e}$ of $G / K$, there exists an open set $O \subseteq G / K$ which contains exactly one of the points $\bar{x}, \bar{e}$. Take $x \in G$ such that $\pi(x)=\bar{x}$. Then $x \notin K$, so there exists an open neighborhood $U$ of $e$ in $G$ such that $x \notin U \cap U^{-1}$ (otherwise $x \in K$ ). If $x \notin U$, then $O=\pi(U)$ is an open neighborhood of $\bar{e}$ in $G / K$ which does not contain $\bar{x}$. If $x \notin U^{-1}$, then $e \notin x U$ and, hence, $O=\pi(x U)$ is an open neighborhood of $\bar{x}$ which does not contain $\bar{e}$.

Let $V$ be a $C$-compact neighborhood of the identity in $G$. Then $W=\pi(V)$ is a $C$-compact neighborhood of $\bar{e}$ in $G / K$, so the group $G / K$ is also locally $C$-compact. Since $G / K$ is a $T_{0}$-space, [29, Lemma 1 and Theorem 1] imply that the restriction of the left quasi-uniformity of $G / K$ to $W \times W$ is hereditarily precompact. Hence, by [19, Theorem 2], $G / K$ is a topological group. Clearly, $K$ inherits the antidiscrete topology from $G$, so $K$ is also a topological group. Therefore, by [26, Lemma 4], $G$ is a topological group as well.

Finally, the local pseudocompactness of $G$ follows from the definition of (local) C-compactness.

\section{$3 \mathbb{R}$-factorizability of feebly compact paratopological groups}

Following [32] we say that a paratopological group $G$ is $\mathbb{R}_{i}$-factorizable for $i=$ $0,1,2,3$ if for every continuous real-valued function $g$ on $G$ one can find a continuous homomorphism $\pi: G \rightarrow H$ onto a second countable paratopological group $H$ satisfying the $T_{i}$ separation axiom and a continuous real-valued function $h$ on $H$ such that $g=h \circ \pi$.

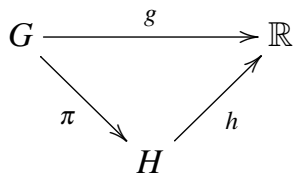

In the next theorem we do not require that the group $G$ satisfy any separation axiom.

Theorem 5 Every feebly compact paratopological group $G$ is $\mathbb{R}_{3}$-factorizable. Furthermore, for every continuous real-valued function $g$ on $G$, there exist a continuous 
homomorphism $\varphi$ of $G$ onto a second countable Hausdorff topological group $H$ and a continuous function $h$ on $H$ such that $g=h \circ \varphi$.

Proof Let $\tau$ be the topology of a feebly compact paratopological group $G$. Denote by $\tau_{r}$ the regularization of the topology $\tau$ and let $G_{r}=\left(G, \tau_{r}\right)$. It is clear that $\tau_{r}$ is coarser than $\tau$. By [23, Example 1.9], $G_{r}$ with the same multiplication is a paratopological group. Therefore, according to [23, Proposition 1.5], the paratopological group $G_{r}$ satisfies the $T_{3}$ separation axiom (however, $G_{r}$ can fail to be even a $T_{0}$-space). Since the topology $\tau_{r}$ is coarser than $\tau$, the group $G_{r}$ is feebly compact. Applying [25, Lemma 6], we conclude that $G_{r}$ is a topological group.

Let $g$ be a continuous real-valued function on $G$. Then $g$ is continuous on $G_{r}$ since the space $\mathbb{R}$ is regular. Indeed, take an arbitrary point $x \in G_{r}$ and an open neighborhood $U$ of $g(x)$ in $\mathbb{R}$. There exists an open neighborhood $V$ of $g(x)$ in $\mathbb{R}$ such that $\bar{V} \subseteq U$. Since $g$ is continuous, we can find an open neighborhood $O$ of $x$ in $G$ such that $g(O) \subseteq V$. Then $W=\operatorname{Int}_{G} \bar{O}$ (the closure is taken in $G$ ) is an open neighborhood of $x$ in $G_{r}$ and

$$
g(W) \subseteq g(\bar{O}) \subseteq \overline{g(O)} \subseteq \bar{V} \subseteq U .
$$

This proves the continuity of $g$ on $G_{r}$. The same function $g$ considered on the space $G_{r}$ will be denoted by $g_{r}$.

Denote by $K$ the closure of the set $\{e\}$ in $G_{r}$, where $e$ is the identity element in $G_{r}$. We claim that the function $g_{r}$ is constant on each coset of $K$ in $G_{r}$. Indeed, take an arbitrary element $x \in G_{r}$. Since $G_{r}$ is a topological group, the closure of the set $\{x\}$ in $G_{r}$ coincides with $x K$. The continuity of the function $g_{r}: G_{r} \rightarrow \mathbb{R}$ implies that $g_{r}(x K) \subseteq\left\{g_{r}(x)\right\}=\left\{g_{r}(x)\right\}$, i.e., $g_{r}$ is constant on $x K$.

Let $p: G_{r} \rightarrow G_{r} / K$ be the quotient homomorphism. Then the quotient group $L=G_{r} / K$ satisfies the $T_{1}$ separation axiom and therefore $L$ is a completely regular topological group. Clearly $L$ is feebly compact as a continuous homomorphic image of $G_{r}$. Hence $L$ is pseudocompact. Since $g_{r}$ is constant on the cosets of $K$ in $G_{r}$, there exists a real-valued function $f$ on the group $L$ satisfying $g_{r}=f \circ p$. The function $f$ is continuous because the homomorphism $p$ is continuous and open.

Since every pseudocompact topological group is precompact and precompact groups are $\mathbb{R}$-factorizable (see [3, Corollary 8.1.17]), we see that the group $L$ is $\mathbb{R}$-factorizable. Hence we can find a continuous homomorphism $\pi: L \rightarrow H$ onto a second countable topological group $H$ and a continuous real-valued function $h$ on $H$ such that $f=h \circ \pi$. Let $i: G \rightarrow G_{r}$ be the identity mapping.

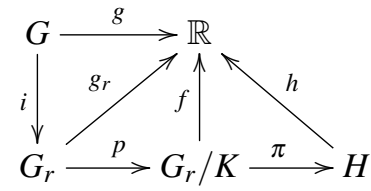

Then the continuous homomorphism $\varphi=\pi \circ p \circ i$ of $G$ onto the second countable topological group $H$ and the function $h$ satisfy the equality $g=h \circ \varphi$. Since $H$ is regular, the group $G$ is $\mathbb{R}_{3}$-factorizable. 
It is worth noting that unlike in topological groups, Theorem 5 is not valid for precompact paratopological groups. Indeed, the circle group $\mathbb{T}_{\text {Sor }}$ endowed with the Sorgenfrey topology is precompact but it is not even $\mathbb{R}_{1}$-factorizable, see [32, Example 3.3]. Another relevant peculiarity of paratopological groups is that feebly compact Hausdorff paratopological groups need not be precompact (see Theorem 1), while every feebly compact topological group satisfying the $T_{0}$ separation axiom is completely regular, pseudocompact, and hence precompact by [10, Theorem 1.1]. In fact, a feebly compact (not necessarily Hausdorff or even $T_{0}$ ) paratopological group $G$ is a topological group iff $G$ is precompact iff $G$ is regular. Indeed, by [2, Theorem 1.7], every regular feebly compact paratopological group is a topological group. Further, by [24, Proposition 3.1], every precompact paratopological group is saturated, while saturated feebly compact paratopological groups are topological groups according to [25, Proposition 2].

Added in proof. a) Recently O. Ravsky succeeded in proving that every 2-pseudocompact paratopological group is feebly compact. Therefore, the feeble compactness of the paratopological groups $G$ and $H$ in Theorems 1 and 3, respectively, can alternatively be deduced from this fact.

b) The second listed author has constructed a precompact completely regular paratopological group $K$ of cardinality $\mathfrak{c}^{+}$such that all subsets of the group $K$ of cardinality less than or equal to $\mathfrak{c}$ are closed. This solves Problem 1 in the affirmative. O. Ravsky proved that all regular 2-pseudocompact paratopological groups are topological groups, thus solving Problem 2.

Acknowledgements The authors are grateful to O. Ravsky for a number of corrections and useful comments about the first version of the article.

\section{References}

1. Alas, O. T., Sanchis, M.: Countably compact paratopological groups. Semigroup Forum 74, 423-438 (2007)

2. Arhangel'skii, A. V., Reznichenko, E. A.: Paratopological and semitopological groups versus topological groups. Topol. Appl. 151, 107-119 (2005)

3. Arhangel'skii, A. V., Tkachenko, M. G.: Topological Groups and Related Structures. Atlantis Studies in Mathematics, Vol. I, Atlantis Press/World Scientific, Paris-Amsterdam, 781 pp. (2008)

4. Banakh, T., Ravsky, A.: On subgroups of saturated or totally bounded paratopological groups. Algebra and Discrete Mathematics, no. 4, pp. 1-20 (2003)

5. Banakh, T., Ravsky, A.: Oscillator topologies on a paratopological group and related number invariants. http://arxiv.org/pdf/0810.3028 (October 16, 2008).

6. Blasco, J. L., Sanchis, M.: On the product of two $b_{f}$-spaces. Acta Math. Hungar. 62, 111-118 (1993)

7. Bokalo, B. M., Guran, I. I.: Sequentially compact Hausdorff cancellative semigroup is a topological group. Mat. Stud. 6, 39-40 (1996)

8. Bouziad, A.: Every Čech-analytic Baire semitopological group is a topological group. Proc. Amer. Math. Soc. 124, 953-959 (1996)

9. Brand, N.: Another note on the continuity of the inverse. Arch. Math. 39, 241-245 (1982)

10. Comfort, W. W., Ross, K. A.: Pseudocompactness and uniform continuity in topological groups. Pacific J. Math. 16, 483-496 (1966)

11. Ellis, R.: A note on the continuity of the inverse. Proc. Amer. Math. Soc. 8, 372-373 (1957)

12. Engelking, R.: General Topology. Heldermann Verlag, Berlin (1989)

13. Frolík, Z:: The topological product of two pseudocompact spaces. Czech. Math. J. 10, 339-349 (1960) 
14. García-Ferreira, S., Romaguera, S., Sanchis, M.: Bounded subsets and Grothendieck's theorem for bispaces. Houston J. Math. 25, 267-283 (1999)

15. Grant, D. L.: Sequentially compact cancellative topological semigroups: some progress on the Wallace problem. In: Papers on General Topology and Applicationa, VIIth Summer Conference at the University of Wisconsin (Madison, 1991), Annals New York Acad. Sci. 704, 150-154 (1993)

16. Hernández, S., Sanchis, M., Tkachenko, M.: Bounded sets in spaces and topological groups. Topology Appl. 101, 21-43 (2000)

17. Katĕtov, M.: On $H$-closed extensions of topological spaces. C̆asopis Pěst. Mat. Fys. 72, 17-32 (1947)

18. Kenderov, P. S., Kortezov, I. S., Moors, W. B.: Topological games and topological groups. Topol. Appl. 109, 157-165 (2001)

19. Künzi, H. P. A., Romaguera, S., Sipacheva, O. V.: The Doitchinov completion of a regular paratopological group. Serdica Math. J. 24, 73-78 (1998)

20. Montgomery, D.: Continuity in topological groups. Bull. Amer. Math. Soc. 42, 879-882 (1936)

21. Numakura, K.: On bicompact semigroups. Math. J. Okayama Univ. 1, 99-108 (1952)

22. Protasov, I. V.: Discrete subsets of topological groups. Math. Notes 55, 101-102 (1994). Russian original in: Mat. Zametki 55, 150-151 (1994)

23. Ravsky, O. V.: Paratopological groups II. Mat. Studii 17, 93-101 (2001)

24. Ravsky, O. V.: The topological and algebraical properties of paratopological groups. Ph.D. thesis, Lviv University; in Ukrainian (2003)

25. Ravsky, O. V.: (2-)Pseudocompact paratopological groups that are topological http://arxiv.org/abs/1003.5343 (March 28, 2010).

26. Ravsky, O. V.: On $H$-closed paratopological groups. http://arxiv.org/abs/1003.5377v1 (March 28, 2010)

27. Ravsky, O., Reznichenko, E.: The continuity of inverse in groups. In: Internat. Conf. Funct. Analysis Appl., Dedicated to the 110th anniversary of Stefan Banach (May 28-31, 2002), Lviv; pp. 170-172 (2002)

28. Reznichenko, E. A.: Extensions of functions defined on products of pseudocompact spaces and continuity of the inverse in pseudocompact groups. Topol. Appl. 59, 233-244 (1994)

29. Romaguera, S., Sanchis, M.: Totally bounded quasi-uniformities and pseudocompactness of bispaces. Monatsh. Math. 138, 289-305 (2003)

30. Romaguera, S., Sanchis, M.: Continuity of the inverse in pseudocompact paratopological groups Algebra Colloq. 14, 167-175 (2007)

31. Romaguera, S., Sanchis, M., Tkachenko, M.: Free paratopological groups. Topol. Proc. 27, 613-641 (2003)

32. Sanchis, M., Tkachenko, M.: $\mathbb{R}$-factorizable paratopological groups. Topology Appl. 157, 800-808 (2010)

33. Stone, M. H.: Applications of the theory of Boolean rings to general topology. Trans. Amer. Math. Soc. 41, 375-481 (1937)

34. Tkachenko, M. G.: Compactness type properties in topological groups. Czech. Math. J. 38, 324-341 (1988)

35. Vaughan, J. E.: On Frolík's characterizations of the class P. Czech Math. J. 44, 1-6 (1994)

36. Żelazko, W.: A theorem on $B_{0}$ division algebras. Bull. Acad. Polon. Sci. Sr. Sci. Math. Astronom. Phys. 8, 373-375 (1960) 